\title{
Lateral Shift of the Femoral Condyle After Total Knee Arthroplasty: Simulation Using 2d-templates of the Medial Pivot Design on Knee Radiographs of Young Japanese Patients
}

\section{Arata Nakajima ( $\nabla$ arata.nakajima@med.toho-u.ac.jp )}

Toho Daigaku Iryo Center Sakura Byoin

\section{Masato Sonobe}

Toho University Sakura Medical Center Department of Orthopaedic Surgery: Toho Daigaku Iryo Center Sakura Byoin Seikei Geka

\section{Yorikazu Akatsu}

Toho University Sakura Medical Center Department of Orthopaedic Surgery: Toho Daigaku Iryo Center Sakura Byoin Seikei Geka

\section{Manabu Yamada}

Toho University Sakura Medical Center Department of Orthopaedic Surgery: Toho Daigaku Iryo Center

Sakura Byoin Seikei Geka

\section{Keiichiro Yamamoto}

Toho University Sakura Medical Center Department of Orthopaedic Surgery: Toho Daigaku Iryo Center Sakura Byoin Seikei Geka

\section{Junya Saito}

Toho University Sakura Medical Center Department of Orthopaedic Surgery: Toho Daigaku Iryo Center Sakura Byoin Seikei Geka

\section{Masaki Norimoto}

Toho University Sakura Medical Center Department of Orthopaedic Surgery: Toho Daigaku Iryo Center Sakura Byoin Seikei Geka

\section{Keita Koyama}

Toho University Sakura Medical Center Department of Orthopaedic Surgery: Toho Daigaku Iryo Center Sakura Byoin Seikei Geka

\section{Shinji Taniguchi}

Toho University Sakura Medical Center Department of Orthopaedic Surgery: Toho Daigaku Iryo Center Sakura Byoin Seikei Geka

Hiroshi Takahashi

University of Tsukuba Faculty of Medicine: Tsukuba Daigaku Igaku Iryokei

\section{Yasuchika Aoki}

Chiba University: Chiba Daigaku 


\section{Toru Suguro}

Toho University: Toho Daigaku

\section{Koichi Nakagawa}

Toho University Sakura Medical Center Department of Orthopaedic Surgery: Toho Daigaku Iryo Center Sakura Byoin Seikei Geka

\section{Research Article}

Keywords: lateral shift, femoral condyle, total knee arthroplasty, simulation, medial pivot design

Posted Date: June 10th, 2021

DOI: https://doi.org/10.21203/rs.3.rs-592112/v1

License: (c) (i) This work is licensed under a Creative Commons Attribution 4.0 International License.

Read Full License 


\section{Abstract}

Background: Total knee arthroplasty (TKA) is an established surgical treatment for advanced knee osteoarthritis by which patients can expect improvement of knee pain and function. Although many surgeons have investigated limb alignment after TKA, changes in coronal positional relation between the femur and tibia are not known well.

Methods: Radiographs of 105 knees of young patients without osteoarthritic changes who received arthroscopic surgeries at our hospital were used in this study. Using 2D-templates of the medial pivot design (the FINE total knee), we simulated TKA on the knee radiographs. First, the femoral component was placed in normal knee alignment and then was merged to the medial concave of the insert where the tibial component was placed in neutral alignment. The length of the mediolateral shift of the femoral component was measured as an estimate of lateral shift of the femoral condyle, of which association with radiographic parameters for the knee was analyzed. Subjects were classified into three groups according to the femoral component size that was chosen in simulation of TKA.

Results: The estimated mean lateral shift of the femoral condyle was $5.99 \pm 1.98 \mathrm{~mm}$ and was greater in males than females $(p<0.05)$. Also, it was most highly correlated with the medial proximal tibial angle (MPTA) $(r=-0.553, p<0.01)$. A group receiving larger component sizes significantly shifted more laterally compared with a group receiving smaller component sizes $(p<0.01)$.

Conclusions: These results suggest that the coronal positional relation between the femur and tibia is altered and subsequent ligament imbalance may occur after mechanically aligned TKA using the medial pivot design.

\section{Background}

The outcomes of total knee arthroplasty (TKA) are generally acceptable; however, approximately $20 \%$ of patients have some complaints after TKA [1-3]. The reasons for dissatisfaction after TKA remain poorly understood; however, one of the causative factors may be changes in coronal positional relation between the femur and tibia after TKA. In TKA using the medial pivot design, femoral components are restricted to the position of tibial inserts, which alters the normal positional relation between the femur and tibia. This may affect balance of major ligaments around the knee including medial collateral ligament (MCL), posterior cruciate ligament ( $\mathrm{PCL}$ ) (if the cruciate ligament-retaining type was used), lateral collateral ligament (LCL), and iliotibial tract (ITT).

The literature states that a neutrally aligned knee has a joint line that is oriented $3^{\circ}$ obliquely (alignment of $3^{\circ}$ varus in the tibia and $3^{\circ}$ valgus in the femur) $[4,5]$. Nowadays, $22 \%$ of men and $17 \%$ of women among asymptomatic adult volunteers between 20 and 27 years-old have constitutional varus knees with a natural mechanical alignment of $3^{\circ}$ varus or more [6]. Restoration of mechanical alignment to neutral in these cases may not be desirable and would be unnatural. 
The FINE total knee has characteristic design features including an oblique $3^{\circ}$ femorotibial joint line. The design of the polyethylene insert includes a medial convex curve with increased conformity to the femoral component, and a lateral flat surface. These features allow internal rotation of the tibia and femoral rollback via medial pivot motion $[7,8]$. However, there are concerns whether the increased medial conformity of the insert may alter the positional relation between the femur and tibia, which would affect the ligament balance.

Many investigators have paid attention to the coronal limb alignment of the hip-knee-ankle angle [1, 918]; however, to the best of our knowledge, there have been few reports looking at changes in coronal positional relation between the femur and tibia after TKA. In this study, using 2D-templates of the medial pivot design, we simulated TKA on knee radiographs of young Japanese patients without osteoarthritic changes. Our hypothesis was that the femoral condyle would be shifted laterally compared to its coronal position in normal alignment.

\section{Methods}

\section{Patients}

Radiographs of 105 knees without osteoarthritic changes were used. Patients were recruited from those between 20 and 49 years-old who received arthroscopic surgeries at our hospital between Jan 2018 and Dec 2020. There were 60 men and 45 women. Demographic data of the patients are shown in Table 1. The diseases included 58 anterior cruciate ligament (ACL) injuries, 13 medial meniscus tears, 11 lateral meniscus tears, seven plica synovialis, six PCL injuries, four cartilage injuries, two patella dislocations, two synovial tumors, one free body and one MCL injury.

This study was approved by the institutional review board at our institution. All activities were performed in accordance with the ethical standards set forth in the Declaration of Helsinki, and informed consent was obtained from all patients who participated.

\section{Measurement of radiographic parameters of the knee}

The femorotibial angle (FTA), lateral distal femoral angle (LDFA), and medial proximal tibial angle (MPTA) were measured on the anteroposterior view of the knee radiographs according to our previous study [19].

\section{Simulation of TKA using 2D-templates on the knee radiographs and measurement of the lateral shift of the femoral condyle}

We applied the 2D-templates of the FINE total knee (Teijin-Nakashima Medical, Okayama, Japan) to the anteroposterior view of the knee radiographs. First, the femoral component was placed to reproduce the form of the distal femoral condyles of each patient and not to overhang (Fig $1 \mathrm{~A}$, green). The tibial component was placed in neutral alignment with the lateral joint line of the minimum thickness $(7 \mathrm{~mm})$ 
of the insert reproducing the original joint line. The size of the femoral and tibial components was chosen to be the same. Then we placed the femoral component so it merged with the medial concave of the tibial insert (Fig 1B, red), which was supposed to be the postoperative alignment. The length of the mediolateral shift of the femoral component was measured and used as an estimate of the lateral shift of the femoral condyle (Fig 1C, a yellow bar).

Three independent observers (AN, MS, YoA) measured the lateral shift of the femoral component. All measurements were performed using a SYNAPSE-PACS software (FUJIFILM, Tokyo, Japan).

\section{Classification for groups of the femoral component size}

The FINE knee has eight size variations of the femoral component ( $S, S+, M, M+, L, L+, 2 L$, and $3 L$ ). Among them, 2D-templates for seven sizes except for $L+$ were available in the software. The sizes were classified into three groups: group 1, S, S+, and M; group 2, M+ and L; group 3, 2L and 3L.

\section{Statistical analyses}

The reliability of each radiographic measurement was assessed using inter- and intra-class correlation coefficients. All radiographic measurements in this study showed good reliability (all values $>0.8$ ).

Significance between groups was analyzed using the t-test or one-factor ANOVA. Results were expressed as the mean \pm standard deviation (SD). Correlations between age, FTA, LDFA, MPTA, and the estimated lateral shift of the femoral condyle were statistically analyzed using the Pearson's correlation coefficient. Data analyses were performed using SPSS software, version 21 (SPSS Inc., Chicago, IL, USA) and $p$ values $<0.05$ were considered statistically significant.

\section{Results}

\section{Radiographic parameters of the knee in young Japanese patients}

The mean FTA, LDFA, and MPTA for male and female subjects are shown in Table 1. The LDFA and MPTA were not statistically different between males and females, while the FTA was significantly greater in males than in females $(p<0.05)$.

\section{Comparison of estimated lateral shift of the femoral condyle between males and females}

The estimated mean lateral shift of the femoral condyle for male and female subjects was $6.41 \pm 1.95$ $\mathrm{mm}$ and $5.43 \pm 1.90 \mathrm{~mm}$, respectively (Table 2). Males had a greater lateral shift of the femoral component than females $(p<0.05)$.

Correlations between estimated lateral shift of the femoral condyle and the radiographic parameters 
Correlation coefficients among radiographic parameters are shown in Table 3. FTA was significantly correlated with LDFA $(r=0.505, p<0.01)$ and MPTA $(r=-0.355, p<0.01)$. There was a significant correlation between LDFA and MPTA $(r=0.309, p<0.01)$. The lateral shift of the femoral condyle had the greatest correlation with MPTA $(r=-0.553, p<0.01)$, and was weakly correlated with LDFA $(r=-0.215, p<$ $0.05)$ and FTA $(r=0.220, p<0.05)$ (Fig. 2$)$.

\section{Comparison of the lateral shift of the femoral condyle between its size groups}

The estimated mean lateral shift for all cases was $5.99 \pm 1.98 \mathrm{~mm}$. The mean lateral shift was $5.24 \pm 1.84$ $\mathrm{mm}$ for group 1, $5.99 \pm 1.78 \mathrm{~mm}$ for group 2, and $6.56 \pm 2.02 \mathrm{~mm}$ for group 3 (Fig. 3). Group 3 significantly shifted more laterally compared with group $1(p<0.01)$, and the mean difference was $1.31 \pm$ $0.42 \mathrm{~mm}$. There was no significant difference in the lateral shift between groups 2 and 3 .

\section{Discussion}

In this study, we demonstrated that the femoral condyle was shifted laterally by an average of $5.99 \mathrm{~mm}$ relative to its position where the implant with the medial pivot design was tentatively placed in normal alignment. Interestingly, the lateral shift was significantly correlated with the MPTA $(r=-0.553)$. According to Bellemans et al., approximately $20 \%$ of young adult volunteers have constitutional varus knees with a natural mechanical alignment of $3^{\circ}$ varus or more [6]. These observations suggest that the lateral shift of the femoral condyle will be greater when patients with constitutional varus alignment receive TKA with the medial pivot design. The lateral shift of the femoral condyle alters the coronal positional relation between the femur and tibia, which may affect balance of ligaments including MCL, PCL, LCL and ITT. Imbalance of those ligaments may cause inappropriate tension of ligaments during knee motion and lead to poor clinical outcomes.

As most patients receiving TKA, except for a bi-cruciate ligament-retaining type, lack ACL function, the tibial insert has a medial concave design to increase stability and allows medial pivot motion. However, this study shows that the medial concave of the insert restricts the position of the femoral component and shifts laterally after TKA. Those are concerns in TKA with the medial pivot design. Clinical studies comparing flat vs. concave inserts have been made and reported [20, 21]. Uvehammer et al. demonstrated that the Hospital for Special Surgery knee score and the patients' opinion based on their preoperative expectations were not very different [20]. On the other hand, a two-year matched pair cohort study demonstrated that the clinical outcomes of the medial pivot design used in CR-TKA were more favorable than those of the flat surface design [21]. It is difficult to draw conclusions about which is better, but the absence of significant differences in clinical scores shown by Uvehammer et al. suggests that even the flat surface allows medial pivot motion when appropriate ligament balances are achieved.

The current study clearly showed that the lateral shift of the femoral condyle was associated with the MPTA in simulations of TKA from the young Japanese knee radiographs. To maintain the normal positional relation between the femur and tibia after TKA, it is important to consider pre-arthritic 
alignment of each patient [22-25]. To date, studies have reported anatomical variations between the femur and tibia in young adult knees [10-12, 14-18, 26]. In a report including 23 Japanese and 47 Caucasian healthy young subjects, Japanese subjects had a significantly greater varus alignment than Caucasians, while women exhibited a more valgus alignment than men [16]. Racial and gender differences in knee-joint obliquity also were reported by Tang et al. Compared with Caucasian subjects, Chinese subjects had significantly larger medial inclination of the knee joint (knee-joint obliquity) and female Chinese subjects had significantly more varus alignment than female Caucasians [18]. Furthermore, Hirschmann et al., using 3D-reconstructed CT scans, demonstrated that there was variability of knee phenotypes in young non-osteoarthritic knees. They showed that in males, the most frequent combination (knee phenotype) was a neutral phenotype in the femur and a neutral phenotype in the tibia, while in females, it was a neutral femoral phenotype and a valgus tibial phenotype [26]. Taken together, these observations suggest that there are racial and gender differences in normal knee alignment and a more individualized approach will be necessary to achieve better clinical outcomes after TKA.

In terms of coronal lower limb alignment in Japanese normal knees, Nakano et al. showed that the femoral condylar orientation and tibial plateau inclination in young and middle-aged (15-54 years-old) participants were greater in males than females [27]. For male participants in their study, the tibial plateau inclination was $85.1 \pm 2.4^{\circ}$ in the young and $85.6 \pm 2.1^{\circ}$ in the middle-aged populations. These angles seem to be smaller than the measurements for similar aged populations by Hirschmann et al. who showed that the tibial mechanical angle was $86.7 \pm 2.3^{\circ}$ for males and $88.0 \pm 2.4^{\circ}$ for females [26]. In the current study, the estimated lateral shift of the femoral condyle was greater in males than in females. Taking these findings into account when planning TKA for Japanese patients, individual tibial plateau inclination should be considered to reconstruct pre-arthritic alignment, especially for male patients.

Mediolateral femoral component position also affects patellar shift and femoral roll-back. Studies using fresh cadaver specimens demonstrated that following mediolateral translation of the femoral component, the patella was significantly shifted and tilted in the same directions, while retropatellar pressure was not significantly altered [28]. More than eight years of follow-up of TKA by van de Groes et al. showed that a medialization of $\geq 5 \mathrm{~mm}$ resulted in a significantly lower anterior knee pain score [29]. These results imply that a lateral shift of the femoral condyle increases the risk of patellofemoral problems such as anterior knee pain in the long-term.

This study has some limitations. First, the sample size was relatively small, and the subjects included patients with meniscus or ligament injuries whose knee alignment may not have been completely normal. Second, the lateral shift of the femoral component was measured on radiographs using 2D-templates of only one knee prosthesis (the FINE total knee). As the estimated lateral shift is affected by implant size variations, the lateral shift will change when other prostheses are used in simulations of TKA. Similar studies using 3D-templates are desirable to better understand the positional relation between the femur and tibia. The present data clearly showed that a lateral shift of the femoral condyle would occur after TKA with the medial pivot design, and this may affect ligament balance; however, biomechanical analyses were not undertaken in this study. Biomechanical studies for the ligament balances using 
computational simulation or cadaver specimens will be required to better understand which ligament balance will be affected and whether those ligament imbalances may cause abnormal knee motion.

\section{Conclusions}

The femoral condyle was shifted laterally compared with its coronal position in normal alignment when simulating TKA with the medial pivot design on knee radiographs of young Japanese patients. The lateral shift of the femoral condyle was greater in males than in females and was significantly correlated with the MPTA. Further studies of the positional relation between the femur and tibia or ligament balance that may be affected by the lateral shift of the femoral condyle are required to achieve better clinical outcomes after TKA.

\section{Abbreviations}

TKA: total knee arthroplasty

MCL: medial collateral ligament

LCL: lateral collateral ligament

ACL: anterior cruciate ligament

PCL: posterior cruciate ligament

ITT: iliotibial tract

LDFA: lateral distal femoral angle

MPTA: medial proximal tibial angle

FTA: femorotibial angle

\section{Declarations}

Ethical approval and consent to participate: Approval for the study was received from the Institutional Review Board at Toho University Sakura Medical Center (approval number: S17012), and all patients gave their written consent to participate in this study. All activities were performed in accordance with the ethical standards set forth in the Declaration of Helsinki.

Consent for publication: All patients gave their written consent to publish to report individual patient data.

Availability of supporting data: The datasets used and/or analyzed during the current study are available from the corresponding author on reasonable request. 
Competing interests: The authors declare that they have no competing interest.

Funding: This study was supported by a Grant-in-Aid for Scientific Research of Japan Society for the Promotion of Science (20K09469 to A.N.).

Authors' contributions: AN participated in the design of the study, performed the statistical analysis and drafted the manuscript. MS and MY participated in the design of the study and performed statistical analyses. YoA, KY and HT participated in the design of the study and helped to draft the manuscript. JS, $\mathrm{MN}, \mathrm{KK}$ and YaA collected patients' clinical information and made a part of figures and tables. TS and KN conceived of the study, participated in its design and coordination and helped to revise the manuscript. All authors read and approved the final manuscript.

Acknowledgements: Not applicable

Authors' information: Their institutions and email addresses are written on the title page.

\section{References}

1. Baker PN, van der Meulen JH, Lewsey J, Gregg PJ. National Joint Registry for England and Wales. The role of pain and function in determining patient satisfaction after total knee replacement. Data from the National Joint Registry for England and Wales. J Bone Joint Surg Br. 2007 Jul;89(7):893900 .

2. Clement ND, Macdonald D, Burnett R. Predicting patient satisfaction using the Oxford knee score: where do we draw the line? Arch Orthop Trauma Surg. 2013 May;133(5):689-694.

3. Minns RJ. The role of gait analysis in the management of the knee. Knee. 2005 Jun;12(3):157-62.

4. Moreland JR, Bassett LW, Hanker GJ. Radiographic analysis of the axial alignment of the lower extremity. J Bone Joint Surg Am. 1987 Jun;69(5):745-9.

5. Hsu RW, Himeno S, Coventry MB, Chao EY. Normal axial alignment of the lower extremity and loadbearing distribution at the knee. Clin Orthop Relat Res. 1990 Jun;225:215-27.

6. Bellemans J, Colyn W, Vandenneucker H, Victor J. The Chitranjan Ranawat Award. Is Neutral Mechanical Alignment Normal for All Patients? The Concept of Constitutional Varus. Clin Orthop Relat Res. 2012 Jan;470(1):45-53.

7. Nakajima A, Sonobe M, Akatsu Y, Aoki Y, Takahashi H, Suguro T, Nakagawa K. Association between limb alignment and patient-reported outcomes after total knee arthroplasty using an implant that reproduces anatomical geometry. J Orthop Surg Res. 2018 Dec 17;13(1):320.

8. Nakajima A, Yamada M, Sonobe M, Akatsu Y, Saito M, Yamamoto K, Saito J, Norimoto M, Koyama K, Takahashi H, Aoki Y, Suguro T, Nakagawa K. Three-year clinical and radiological results of a cruciateretaining type of the knee prosthesis with anatomical geometry developed in Japan. BMC Musculoskelet Disord. 2021 Mar;3(1):241. 22(. 
9. Hirschmann MT, Behrend H. Functional knee phenotypes: a call for a more personalized and individualized approach to total knee arthroplasty? Knee Surg Sports Traumatol Arthrosc. 2018 Oct;26(10):2873-2874.

10. Hirschmann MT, Amsler PK, Iranpour F, Friederich NF, Cobb JP. The position and orientation of total knee replacement components: a comparison of conventional radiographs, transverse 2D-CT slices and 3D-CT reconstruction. J Bone Joint Surg Br. 2011 May;93(5):629-33.

11. Jabalameli M, Moghimi J, Yeganeh A, Nojomi M. Parameters of lower extremities alignment view in Iranian adult populations. Acta Med Iran. 2015;53(5):293-6.

12. Kawakami H, Sugano N, Yonenobu K, Yoshikawa H, Ochi T, Hattori A, Suzuki N. Effects of rotation on measurement of lower limb alignment for knee osteotomy. J Orthop Res. 2004 Nov;22(6):1248-53.

13. Lampart M, Behrend H, Moser L, Hirschmann MT. Due to great variability fixed HKS angle for alignment of the distal cut leads to a significant error in coronal TKA orientation. Knee Surg Sports Traumatol Arthrosc. 2019 May;27(5):1434-41.

14. Mullaji AB, Marawar SV, Mittal V. A comparison of coronal plane axial femoral relationships in Asian patients with varus osteoarthritic knees and healthy knees. J Arthroplasty. 2009 Sep;24(6):861-7.

15. Cooke D, Scudamore A, Li J, Wyss U, Bryant T, Costigan P. Axial lower-limb alignment: comparison of knee geometry in normal volunteers and osteoarthritic patients. Osteoarthritis Cartilage. 1997 Jan;5(1):39-47.

16. Hovinga KR, Lerner AL. Anatomical variations between Japanese and Caucasian populations in the healthy young adult knee joint. J Orthop Res. 2009 Sep;27(9):1191-6.

17. Nayak M, Kumar V, Yadav R, Maredupaka S, Srivastava DN, Malhotra R, Pandit H. Coronal alignment of the lower extremity: a gender-based ratio-graphic analysis in Indian patients. Indian J Orthop. 2020 Mar;10(4):504-12. 54(.

18. Tang WM, Zhu YH, Chiu KY. Axial alignment of the lower extremity in Chinese adults. J Bone Joint Surg Am. 2000 Nov;82(11):1603-8.

19. Toguchi K, Nakajima A, Akatsu Y, Sonobe M, Yamada M, Takahashi H, Saito J, Aoki Y, Suguro T, Nakagawa K. Predicting clinical outcomes after total knee arthroplasty from preoperative radiographic factors of the knee osteoarthritis. BMC Musculoskelet Disord. 2020 Jan 6;21(1):9.

20. Uvehammer J, Regnér L, Kärrholm J. Flat vs. concave tibial joint surface in total knee arthroplasty: randomized evaluation of 39 cases using radiostereometry. Acta Orthop Scand. 2001 Jun;72(3):257-65.

21. Nakamura J, Inoue T, Suguro T, Suzuki M, Sasho T, Hagiwara S, Akagi R, Orita S, Inage K, Akazawa T, Ohtori S. A comparative study of flat surface design and medial pivot design in posterior cruciateretaining total knee arthroplasty: a matched pair cohort study of two years. BMC Musculoskelet Disord. 2018 July 18;19(1):234.

22. Eckhoff DG, Bach JM, Spitzer VM, Reinig KD, Bagur MM, Baldini TH, Flannery NM. Three-dimentional mechanics, kinematics, and morphology of the knee viewed in virtual reality. J Bone Joint Surg Am. 2005;87(Suppl 2):71-80. 
23. Howell SM, Howell SJ, Hull ML. Assessment of the radii of the medial and lateral femoral condyles in varus and valgus knees with osteoarthritis. J Bone Joint Surg Am. 2010 Jan;92(1):98-104.

24. Howell SM, Hull ML. Kinematic alignment in total knee arthroplasty. In: Scott WN, editor. Insall and Scott Surgery of the Knee. Philadelphia: Elsevier; 2012. pp. 1255-68.

25. Howell SM, Kuznik K, Hull ML, Siston RA. Results of an initial experience with custom-fit positioning total knee arthroplasty in a series of 48 patients. Orthopedics. 2008 Sep;31(9):857-63.

26. Hirschmann MT, Moser LB, Amsler F, Behrend H, Leclercq V, Hess S. Phenotyping the knee in young non-osteoarthritic knees shows a wide distribution of femoral and tibial coronal alignment. Knee Surg Sports Traumatol Arthrosc. 2019 May;27(5):1385-93.

27. Nakano N, Matsumoto T, Hashimura M, Takayama K, Ishida K, Araki D, Matsushita T, Kuroda R, Kurosaka M. Coronal lower limb alignment in normal knees-A radiographic analysis of 797 normal knee subjects. Knee. 2016 Mar;23(2):209-13.

28. Steinbrück A, Schröder C, Woiczinski M, Schmidutz F, Müller P, Jansson V, Fottner A. Mediolateral femoral component position in TKA significantly alters patella shift and femoral roll-back. Knee Surg Sports Traumatol Arthrosc. 2017 Nov;25(11):3561-8.

29. van de Groes SAW, Koëter S, de Waal Malefit M, Verdonschot N. Effect of medial-lateral malpositioning of the femoral component in total knee arthroplasty on anterior knee pain at greater than 8 years of follow-up. Knee. 2014 Dec;21(6):1258-62.

\section{Tables}

Due to technical limitations, table 1 to 3 is only available as a download in the Supplemental Files section.

\section{Figures}



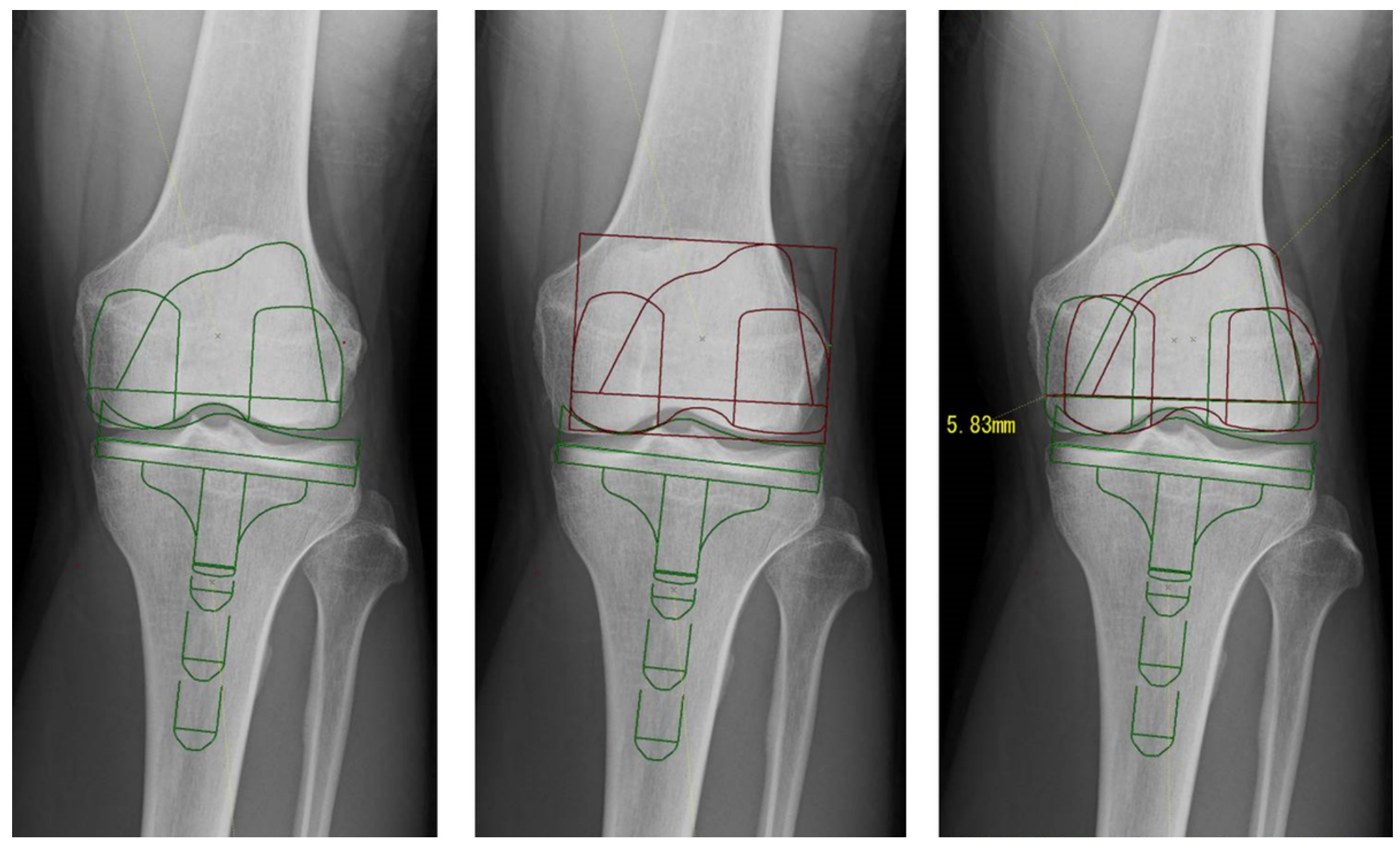

\section{Figure 1}

Simulation of TKA with the medial pivot design (the FINE total knee) on knee radiographs of young patients. The femoral component was placed to reproduce the form of the distal femoral condyles of the patients and not to overhang (A, green). The tibial component was placed in neutral alignment with the lateral joint line of the minimum thickness $(7 \mathrm{~mm})$ of the insert reproducing the original joint line. Then, we placed the femoral component to merge with the medial concave of the tibial insert ( $B$, red). The length of the lateral shift of the femoral component was measured ( $C$, a yellow bar). In this case, the $2 \mathrm{~L}$ size was chosen and the lateral shift was $5.83 \mathrm{~mm}$.

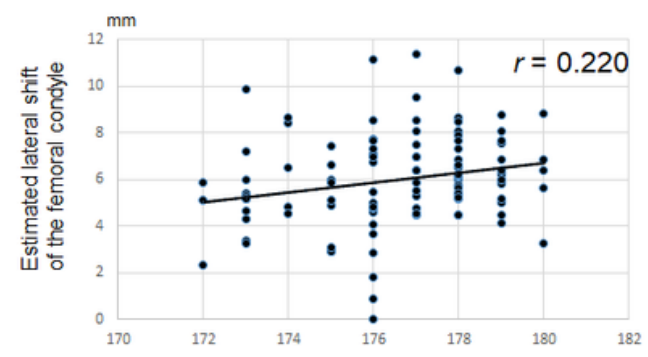

FTA ( $\left.{ }^{\circ}\right)$

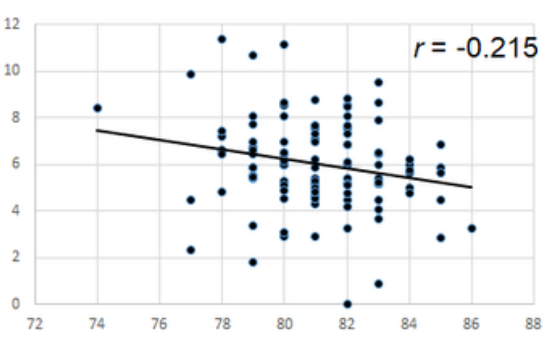

$\operatorname{LDFA}\left({ }^{\circ}\right)$

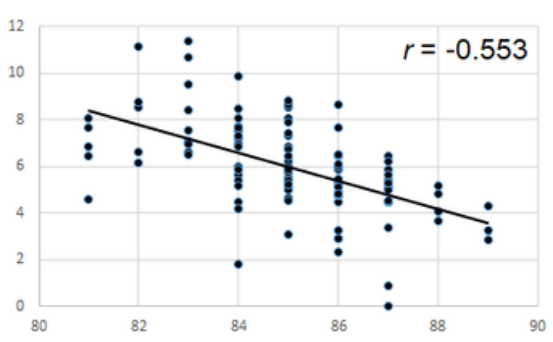

MPTA $\left(^{\circ}\right)$

\section{Figure 2}

Correlations between the estimated lateral shift of the femoral condyle and radiographic parameters of the knee. The lateral shift of the femoral condyle was most highly correlated with MPTA $(r=-0.553)$, and 
weakly correlated with LDFA $(r=-0.215)$ and FTA $(r=0.220)$. MPTA, medial proximal tibial angle; LDFA, lateral distal femoral angle; FTA, femorotibial angle.

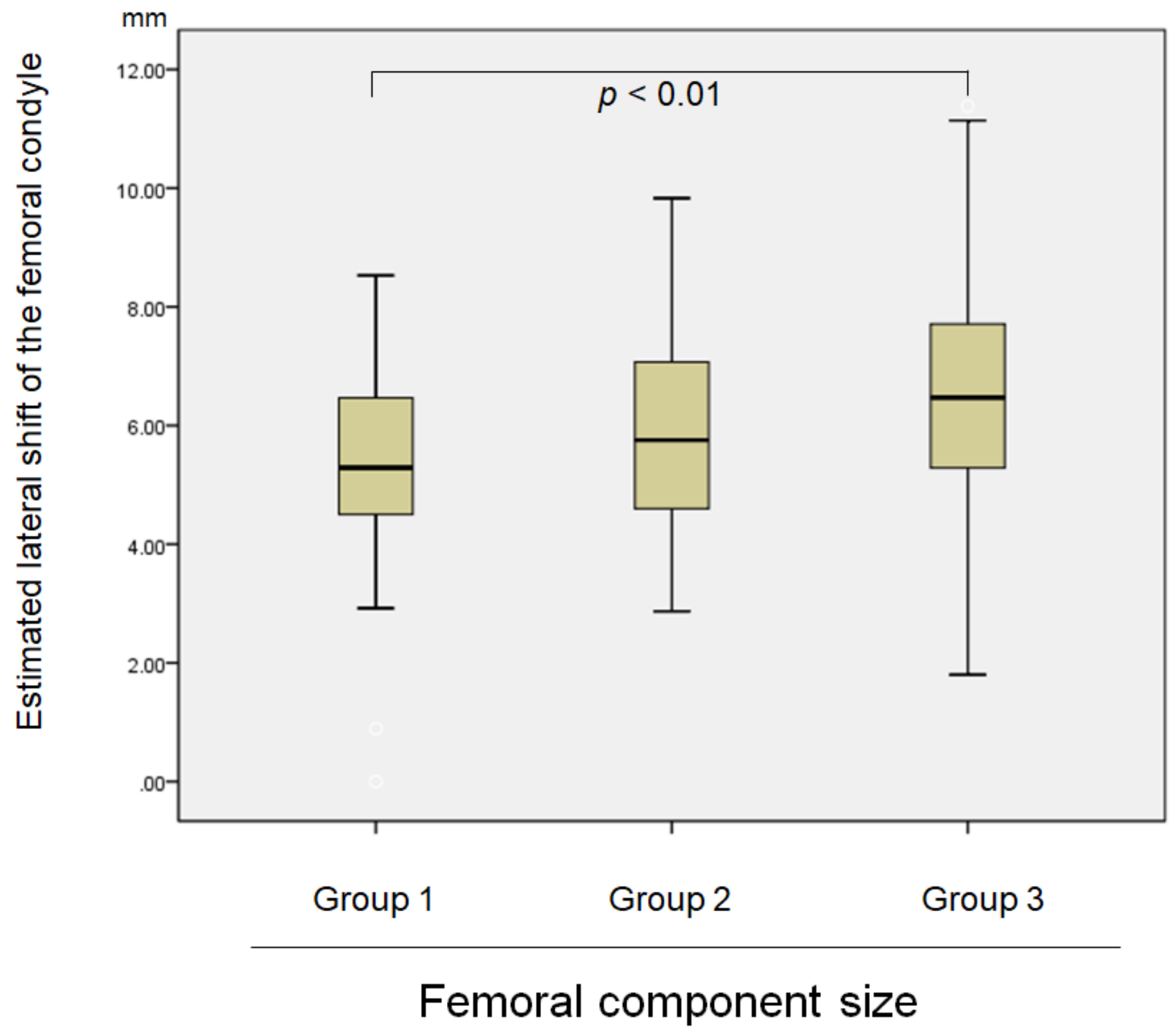

\section{Figure 3}

Comparison of the estimated lateral shift of the femoral condyle between size groups for the FINE knee. Group 1 includes sizes for S, S+ and M; group 2 for $M+$ and $L$; group 3 for $2 \mathrm{~L}$ and $3 \mathrm{~L}$. Group 3 significantly shifted more laterally compared with group $1(\mathrm{p}<0.01)$. Thick horizontal line: median value; box: interquartile range (IQR); whiskers: most extreme points within 1.5 times the IQR from the limits of the box.

\section{Supplementary Files}


This is a list of supplementary files associated with this preprint. Click to download.

- table1.xlsx

- table2.xIsx

- table3.xlsx 